\title{
Receptores de progesterona en meningioma.
}

\author{
Progesterone receptors in meningioma.
}

Ojeda Di Ninno Herminio*, Castro De la Matta, Olga**.

*Médico cirujano, ex - alumno, Universidad Peruana Cayetano Heredia.

**Jefe del Departamento de Inmunohistoquímica. Instituto Nacional de Enfermedades Neoplásicas. Lima, Peru.

\section{RESUMEN}

Objetivo: Determinar la presencia de los receptores de progesterona en meningiomas y su frecuencia mediante la inmunohistoquímica. Material y Métodos: Se analizaron 24 muestras provenientes de pacientes intervenidos quirúrgicamente en el Instituto Nacional de Enfermedades Neoplásicas entre los años 1990 y 1992 con diagnóstico anatomopatológico de meningioma. La determinación de los receptores se hizo mediante una técnica de inmunohistoquímica rápida que permite el estudio de tejidos fijados previamente en parafina. Resultados: De los 24 casos estudiados, nueve resultaron ser positivos en la determinación de receptores de progesterona (37\%). Se pudo observar un marcado predominio dentro del grupo femenino quienes constituyeron 8/9 casos positivos. Conclusiones: El empleo de esta reciente técnica de inmunohistoquímica aplicada a tejido de fijado en parafina, nos ha permitido confirmar la presencia de receptores de progesterona en meningiomas con una frecuencia elevada que creemos amerita un estudio más amplio de manera sistemática que incluya la intervención terapéutica mediante el uso de antiprogestágenos, como el Mifepristone o RU 486. De este estudio podrían beneficiarse no sólo pacientes operados recientemente sino aquellos que, intervenidos en el pasado sean detectados como portadores de receptores de progesterona mediante la aplicación de esta novedosa técnica (Rev Med Hered 1995; 6: 121-130).

PALABRAS CLAVE: Receptor de progesterona, meningioma, inmunohistoquímica.

\section{SUMMARY}

Objective: To determine the presence of the progesterone receptors in meningiomas and its frequency by means of immunohistochemistry. Material and methods: We studied 24 samples coming from patients who have been operated at the Instituto Nacional de Enfermedades Neoplasicas in Lima, Perú, between 1992 and 1992 because of the presence of a cerebral tumor which gave the final diagnosis of meningioma on the pathology report. The determination of the presence of these receptors was done by the immunohistochemical technic on meningioma tissue previously fixed on paraffin. Results: From the 24 cases 
studied we have found nine to be positive to progesterone receptors which represented the thirty seven per cent of the total of meningioma samples analyzed. We have observed a marked predominance in the female group which represented eight of the nine positive cases. Conclusions: The use of this recent developed technic of immunohistochemistry applied to fixed tissue in paraffin has permitted us to confirm the presence of progesterone receptors in meningiomas with an elevated frequency which merits a more ample study in a systematic way which should include a therapeutic approach with the use of antiprogestagens like Mifepristone or RU 486. From this study not only recently operated patients could be beneficiated but also those who have been operated in the past and that could be detected as carriers of progesterone receptors with the application of this new technic. (Rev Med Hered 1995; 6: 121-130).

KEY WORDS: Progesterone receptor, meningioma, immunohistochemistry.

\section{INTRODUCCION}

Los meningiomas son los tumores benignos más frecuentes del sistema nervioso central constituyendo del $15 \%$ al $19 \%(1,2)$, pudiendo ser múltiples hasta en un $8 \%$ (3) y rara vez producen metástasis, como en el caso de los meningosarcomas, siendo los pulmones, hígado y ganglios linfáticos los lugares más comunes $(2,4,5)$.

Usualmente estos tumores se originan a partir de las células aracnoideas (6), crecen lentamente y son extraxiales (7). Existen evidencias que indican a un factor hormonal en su crecimiento, como el hecho de incrementar su tamaño durante el embarazo, la ocurrencia hasta 2 veces más frecuentemente en mujeres que en los varones y su asociación frecuente con el carcinoma mamario (8). Existen importantes publicaciones sobre la existencia de receptores para factores de crecimiento y hormonas $(9,10,11,12,13,14,15,16,17,18)$ como los de estrógenos $(14,18,20,21,22,23)$, D1 dopaminérgicos (4) y especialmente de progesterona en los meningiomas $(10,14,19,21,23-31)$, estos últimos particularmente, podrían estar implicados en el crecimiento tumoral.

Los meningiomas a pesar de su condición de ser tumores benignos no son siempre curables y las tasas de recurrencia varían de acuerdo al grado de resección obtenida, su ubicación y su agresividad biológica (32). Así por ejemplo cuando se logra una extirpación total la tasa de recurrencia esperada a 10 años es del $8 \%$ al $10 \%$ contra $29 \%$ al $44 \%$ en los casos en que queda tumor residual (33). La tasa total de recurrencia general luego de un seguimiento de 20 años va del 19\% al 50\% según algunas series (2). La radioterapia puede constituir una terapia adyuvante. Un estudio demuestra una tasa de recurrencia del $4 \%$ en el caso de remoción quirúrgica completa y de $32 \%$ en los casos en que la resección fue parcial y se usó de manera adyuvante la radioterapia ocurrió en 125 meses en promedio y luego de 66 meses en los casos que no recibieron radioterapia. Si bien esto demostraría un rol beneficioso en los tumores resecados parcialmente, la radioterapia ha sido asociada al desarrollo de astrocitomas malignos al ser usada para meningiomas. De otro lado los efectos adversos de la radioterapia son bien conocidos.

La alternativa actualmente en investigación del uso de hormonoterapia como adyuvante en meningioma $(9,12,29,34-44)$, cobra especial interés dada la frecuencia de esta patología, sus altas tasas de recurrencia y la morbilidad que esta ocasiona por su ubicación y/o difícil resecabilidad en algunos casos. 
El objetivo del presente trabajo fue determinar la presencia de receptores de progesterona en muestras fijadas en parafina correspondientes a meningiomas, mediante las técnicas de inmunohistoquímica en un grupo de pacientes operados en el Servicio de Neurocirugía del Instituto Nacional de Enfermedades Neoplásicas entre los años 1990 y 1992.

\section{MATERIAL Y METODOS}

Veinticuatro muestras tumorales, informadas anatomo-patológicamente como meningioma, fueron consideradas para la determinación de receptores de progesterona mediante análisis inmunohistoquímico. Los pacientes fueron intervenidos entre los años 1990 y 1992 en el Instituto Nacional de Enfermedades Neoplásicas.

Se registraron datos provenientes de las historias clínicas de los referidos pacientes en fichas preparadas especialmente, en donde se registraron edad, sexo, ocupación, lugar de nacimiento y procedencia, así como tiempo de enfermedad, sintomatología principal y si se trataba de un tumor primario o recurrente. Se consignaron igualmente el tipo histológico (26), localización del meningioma, grado de remoción tumoral quirúrgica según Simpson (2) e informe tomográfico de la lesión. Se revisaron antecedentes epidemiológicamente relacionados con la aparición o crecimiento de los meningiomas $(1,7,8,45)$.

Para el estudio inmunohistoquímico se utilizaron anticuerpos monoclonales (Biogenex $($ ) antireceptor nuclear de progesterona de ratón de la clona PGR-1A6 y perteneciente a la clase IgG1 los que fueron diseñados para la detección de los mismo ya sea en tejidos fijados en formalina y embebidos en parafina o en secciones de tejidos congelado $(46,47,48)$ los cuales fueron procesados de acuerdo al protocolo (Cuadro $\mathrm{N}^{\mathrm{o}} 1$ ).

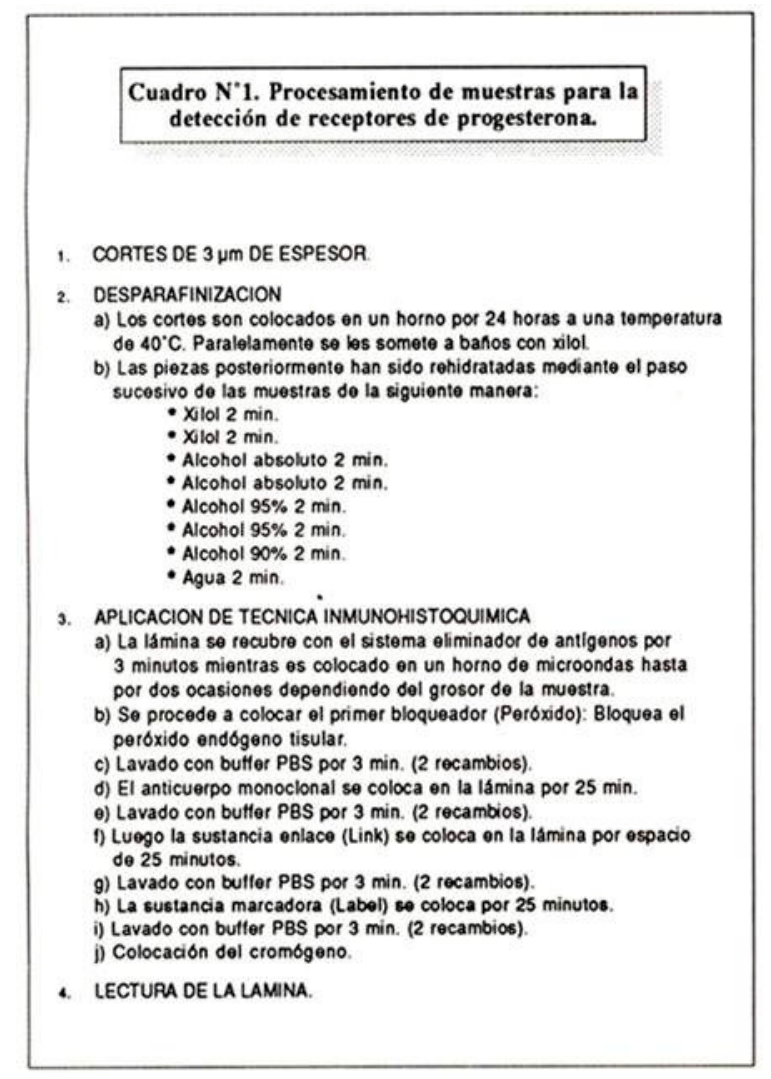


Los anticuerpos monoclonales se presentan diluídos en buffer fosfato salino, $\mathrm{pH} 7.6$ con $1 \%$ de albúmina de suero bovino y siendo su estabilidad de 18 meses a 2-8 C.

La lectura de las láminas estuvo a cargo de una especialista en inmunohistoquímica del Instituto Nacional de Enfermedades Neoplásicas, quien clasificó las reacciones como negativas, de no presentarse tinción nuclear o de positivas de existir coloración marrón de los núcleos, efectuándose luego de una graduación de 1 a 4 cruces de positividad de acuerdo a la intensidad y número de núcleos observados.

\section{RESULTADOS}

La edad promedio de los pacientes estudiados fue de 43 años con un rango entre los 15 y los 73 años, mientras que para el grupo que presentó receptores positivos de progesterona el promedio de edad se encontró en 49 años (rango: 30-73 años). El sexo predominante fue el femenino con el $80 \%$ del total de los casos registrados, constituyendo el $88 \%$ del total de las muestras positivas. El porcentaje de varones y mujeres positivas dentro de su mismo género fue de 20 y $42 \%$ respectivamente.

Del total de nueve casos positivos para receptores de progesterona, ocho correspondieron a mujeres. Tres correspondían al periodo pre menopáusico y cuatro pertenecían al grupo de menopáusicas. En uno de los casos no se encontró los datos correspondientes a los antecedentes ginecológicos.

Ninguno de los pacientes presentó meningioma de carácter recurrente. No existían evidencias de metástasis en ninguno de los casos. El tiempo promedio de enfermedad de los pacientes fue de 36 meses como promedio general y de 51 meses para aquellos positivos a receptores de progesterona. Todos tuvieron ubicación intracraneal y 10/24 pacientes presentaban edema peritumoral demostrado topográficamente (Cuadro $\left.\mathrm{N}^{\circ} 2\right)$.

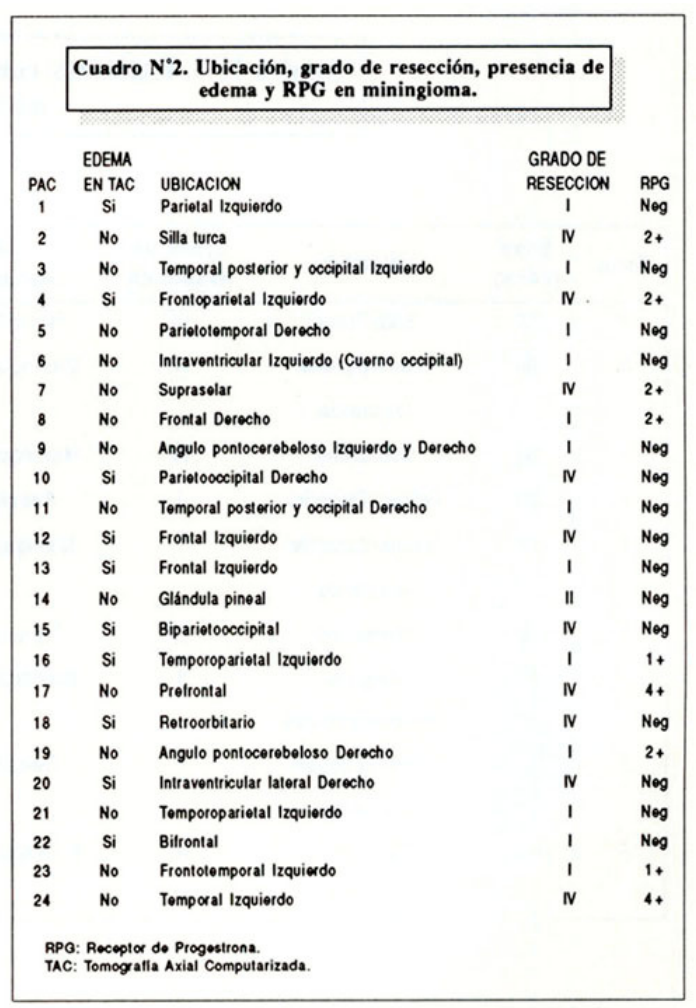


Según el grado de resección tumoral de Simpson (Cuadro No3), se obtuvo que trece de los 24 casos fueron del grado I, diez correspondieron al grado IV y solamente un caso del grado II. De los casos positivos, 4 tuvieron un grado de resección del tumor de grado I y los cinco restantes pertenecieron al grado IV.

\begin{tabular}{|c|c|}
\hline & Cuadro $N^{\circ} 3$. Escala de Simpson. \\
\hline Grado & Grado de Remocion \\
\hline I & $\begin{array}{l}\text { Remoción completa macroscópica con excision de } \\
\text { pedículo dural y hueso anormal. }\end{array}$ \\
\hline II & $\begin{array}{l}\text { Remoción completa macroscópica con coagulación } \\
\text { endodérmica (con laser o bovie) del pedículo dural }\end{array}$ \\
\hline III & $\begin{array}{l}\text { Remoción completa sin remoción o coagulacion del } \\
\text { pedículo dural. }\end{array}$ \\
\hline IV & Remoción parcial del tumor dejando parte in situ. \\
\hline $\mathrm{V}$ & Descompresion simple con o sin biopsia. \\
\hline
\end{tabular}

Del total de los casos estudiados, 17 fueron del tipo de meningoteliomatoso, 3 correspondieron al tipo transicional, 2 fueron de tipo fibroblástico mientras los dos restantes pertenecieron uno al tipo maligno ó anaplásico y el otro al angioblástico. Los que mostraron receptores positivos para progesterona, 5 fueron del tipo meningoteliomatoso, del tipo transicional, uno del tipo anaplásico ó maligno y uno del tipo fibroblástico (Cuadro $\mathrm{N}^{\circ} 4$ ).

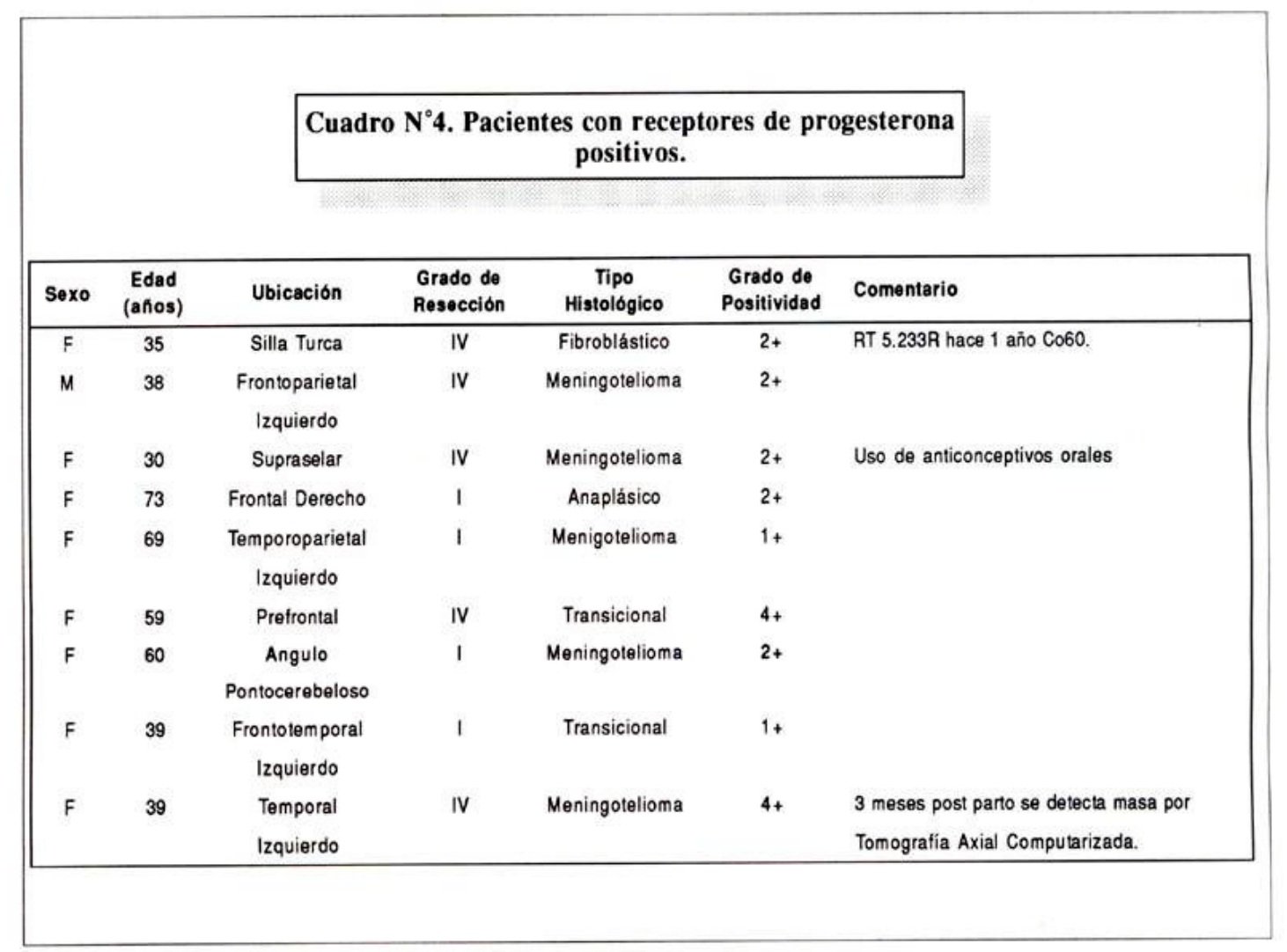


Las microfotografías muestran tanto los casos positivos como los negativos de acuerdo a la técnica de inmunohistoquímica (Figura $\mathrm{N}^{\mathrm{o}} 1$, figura $\mathrm{N}^{\mathrm{0}} 2$, figura $\mathrm{N}^{\circ} 3$ y figura $\mathrm{N}^{\mathrm{o}} 4$ ).

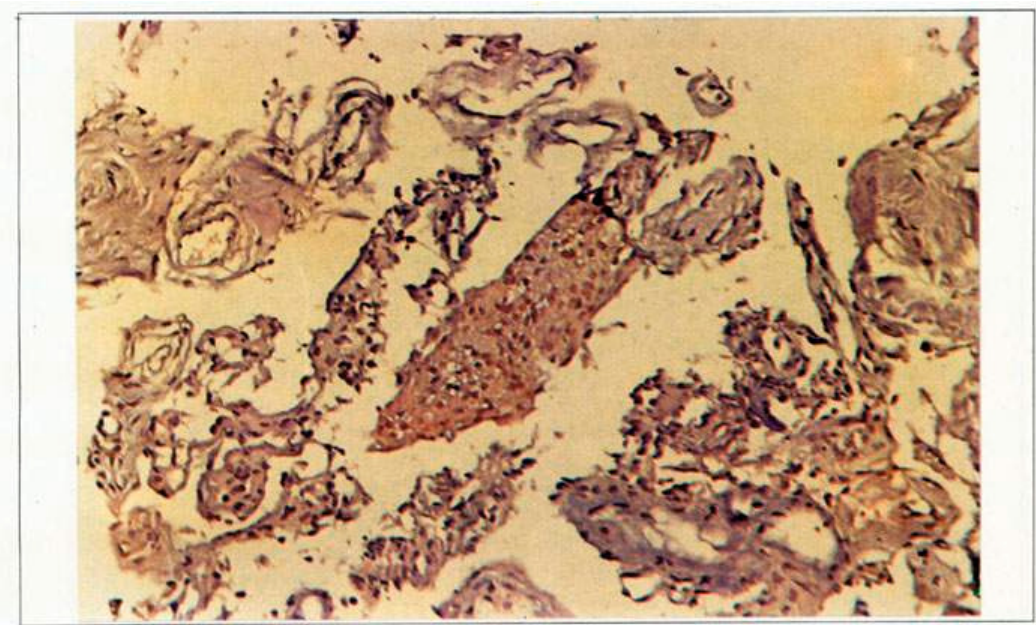

Figura $\mathrm{N}^{\circ} 1$. Meningioma de tipo meningoteliomatoso con estroma mixoide: Los núcleos son fuertemente positivos en las áreas no mixoides $(\mathrm{x} 400)$.

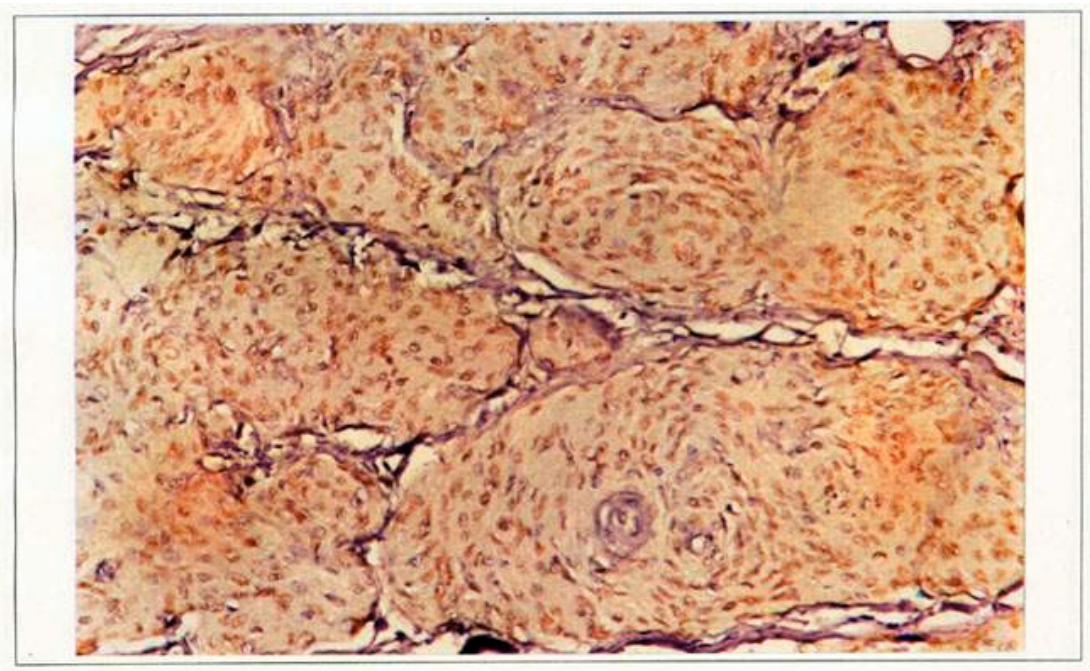

Figura $\mathrm{N}^{\circ}$ 2. Meningioma de tipo meningoteliomatoso altamente vascular: Todos los núcleos son positivos con una intensidad mediana en la reacción nuclear $(\times 400)$.

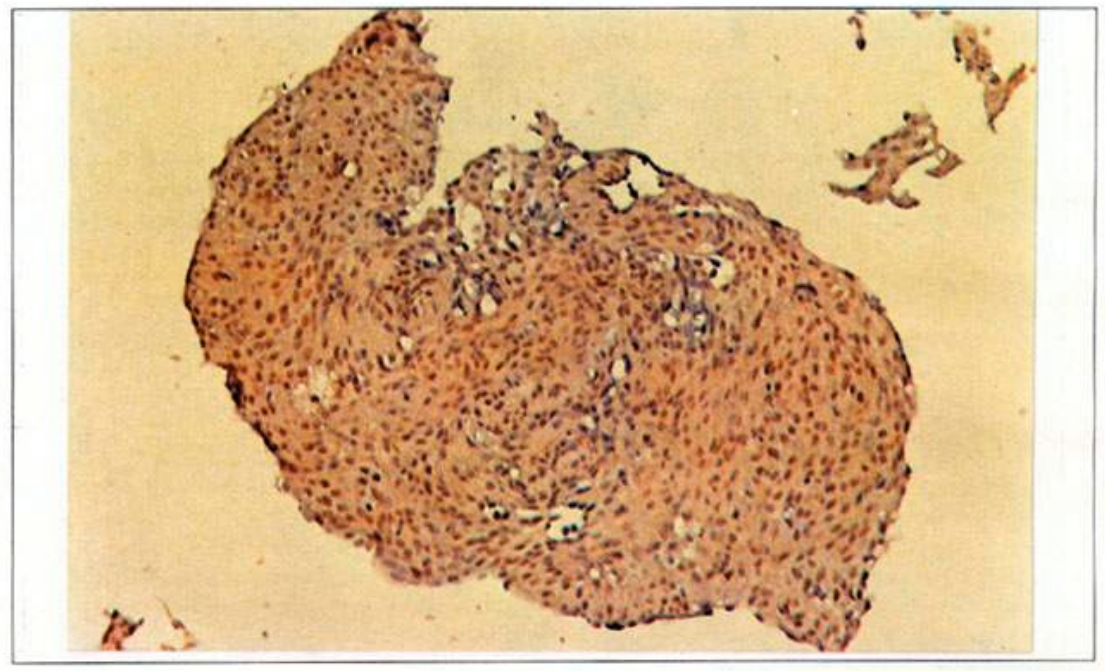

Figura $\mathrm{N}^{\circ}$ 3. Meningioma meningoteliomatoso: No todos los núcleos son positivos y la intensidad de la reacción es variable $(\times 400)$. 


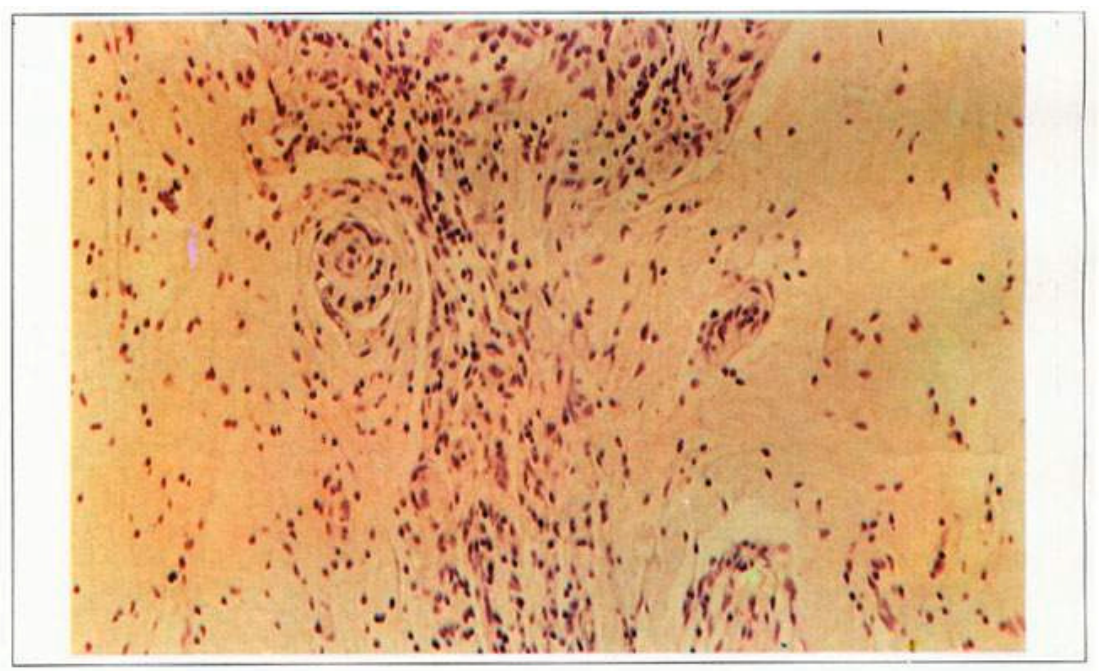

Figura $\mathrm{N}^{\circ} 4$. Meningioma de tipo angioblástico: La reacción es negativa $(x 400)$.

\section{DISCUSION}

Hemos podido demostrar mediante técnicas de inmunohistoquímica la presencia de receptores de progesterona en tejido con diagnóstico anatomopatológico de meningioma previamente fijado en parafina en el $37.5 \%$ de los casos estudiados. Las diferentes series reportan una positividad para la presencia de receptores de progesterona entre el 40 y $93 \%$. Esta variedad en los resultados se puede deber a las diferentes técnicas empleadas $(13,17,26)$ o a lo que se denomina heterogenicidad celular tumoral del meningioma. Así por ejemplo, se sabe y está bien demostrando que en el tejido de carcinoma mamario las células de diferentes partes del mismo tumor varían tanto cuantitativa como cualitativa como cualitativamente $(17,26)$.

La técnica empleada para este trabajo mediante el uso de anticuerpos mononucleares contra receptores de progesterona de fabricación reciente permite la evaluación rápida (aproximadamente en 2 horas) tanto de tejido fresco de meningioma extirpado así como aquél que hacer varios años atrás fue extirpado y debidamente fijado y conservado en parafina a diferencia de técnicas empleadas en estudios previos que requieren tejido reciente, además demuestra la distribución de los receptores en la célula de manera precisa.

Resulta particularmente curioso que siendo el tejido meníngeo de origen mesodermal y que normalmente no presentan receptores para progesterona (17), presenten receptores de carácter progestágeno al transformarse en meningiomas. Como sabemos el hallazgo de receptores de progesterona hasta hace pocos años controversial, es ahora casi universalmente aceptado $(24,26,28,30,31)$ en contraste con lo que acontece con la determinación de los receptores estrogénicos cuya presencia tanto cuantitativa como cualitativamente en meningiomas ha sido poco relevante desde el punto de vista patológico y clínico $(17,26,30,31,49,50)$.

Si bien la mayoría de nuestros pacientes hicieron uso de glucocorticoides preoperatoriamente y este hecho ha sido vinculado a la presencia de receptores de progesterona por algunos como un efecto no específico d los esteroides, esto ya ha sido desvirtuado por la presencia de los mismos en pacientes que no recibieron dexametasona antes de la cirugía 
(17). La afinidad de tipo específica de los receptores hacia hormonas sexuales también ha sido demostrada $(17,31)$.

Podemos afirmar en este trabajo que los receptores encontrados en los meningiomas estudiados son al menos afines con los expresados en el carcinoma mamario ya que los anticuerpos monoclonales usados se aplican de rutina en el estudio de esta última patología. Esta semejanza inmunohistoquímica ya ha sido descrita antes (30) y al menos muy sugerida por otros, inclusive por estudios de síntesis de RNA mensajero para receptor de meningioma $(24,25)$. Como sabemos la asociación temporal entre los meningiomas y el carcinoma de mama está reconocida $(8)$.

Se pudo observar en la muestra estudiada una marcada prevalencia de receptores de progesterona positivos en el sexo femenino constituyendo el $43 \%$ de este grupo contra $20 \%$ del total de varones. Resulta interesante además el hecho que una mujer fuertemente positiva (4+), presentó 60 meses como tiempo de enfermedad y cuyos síntomas se exacerban 3 meses post parto. Como se sabe está reconocido el crecimiento de los meningiomas durante el embarazo (26). No menos interesante es el hecho en otro de los casos en los que una paciente positiva había recibido anticonceptivos orales por 8 años. Estos dos hechos observados en este trabajo al igual que en otros estudios de este tipo así como aquellos de corte epidemiológico sugieren en cierta medida la posibilidad de que estos tumores al menos en parte se vean influenciados en su crecimiento por hormonas sexuales.

La ausencia y/o poca presencia de receptores de estrógenos en los meningiomas demostrada en varios estudios previos y por diferentes técnicas hacen suponer que el efecto de estos sobre la expresión de receptores de progesterona en los meningiomas es escasa $(15,17,19,22,25,38)$ a diferencia del rol que juegan en el carcinoma de mama o tejido uterino en los que los receptores de estrógeno promueven su expresión (26). Como se reporta los receptores hormonales son proteínas intracelulares que juegan un papel importante en la regulación de la expresión genética, la síntesis del DNA así como de proteínas y en el crecimiento celular, lo que ha llevado a algunos autores a realizar estudios in vitro en donde demuestran un papel en la génesis tumoral por parte de estos receptores y que en vivo pueden contribuir a la formación y/o crecimiento de los meningiomas (25).

Cabe señalar que otras hormonas, a parte de la progesterona, al igual de algunos neurotransmisores y factores de crecimiento han sido encontrados en los meningiomas y han sido implicados directa o indirectamente con su crecimiento al menos in vitro (51) como andrógenos (25), somatostatina (52), factor de crecimiento epidermal $(10,11,51,52)$, factor de crecimiento semejante a la insulina (11) y dopamina D1 (4). El rol de las mismas en el desarrollo de meningiomas es sugerente pero aún están en estudio.

El tamaño de la muestra estudiada permite evaluar frecuencia pero no permite determinar diferentas estadísticamente significativas, pero las correlaciones de la presencia o no de receptores con datos epidemiológicos, status reproductivos e inclusive histología tumoral es variable $(15,17,28)$ con cada estudio siendo esto explicado tal vez por la heterogeneidad celular tumoral ya descrita previamente. Desde el punto de vista d imágenes existe un estudio que revela relación entre la presencia de receptores de progesterona en los meningiomas y la ocurrencia de enema peritumoral en la tomografía así como con la existencia de una disrupción de la cortical y el tamaño del tumor. En otro estudio determinan que la presencia de receptores de progesterona, presentaba correlación significativa con la presencia de edema peritumoral mientras otros no lo llegan a demostrar (36,53). En nuestro estudio sólo 10 del total, presentaron edema en la tomografía pero sólo 2 del total de positivos mostraron edema 
en la tomografía.

Algunos autores han encontrado relación entre la presencia de receptores para progesterona con los tipos patológicos correspondiente al transicional y meningoteliomatoso (54), sin embargo nuestro tamaño muestral no nos permite concluir relación alguna con el tipo histológico.

Sabemos que el tratamientote elección en estos momentos para los meningiomas lo constituye la cirugía, en la medida que estos son resecables y el uso de la radioterapia. Con respecto al uso de antiprogestágenos existen actualmente algunas evidencias que favorecen su uso aunque es algo que requiere más estudios. Como sabemos la presencia poco relevante de receptores estrogénicos como ya se destacó en la introducción, aunado al hecho que los receptores progestágenos no son modulados por estos $(40,55)$ no ha motivado el uso de antiestrogénicos como terapia experimental y en los casos que se han utilizado estos no han mostrado efectos relevantes.

En 1985 Philippe Courriere y colaboradores sugieren que la presencia de receptores esteroideos en tumores intracraneales podría abrir una alternativa de terapia endocrina en pacientes de alto riesgo o con resección tumoral incompleta (44). Jay y colaboradores realizan trabajos in vitro y demuestran aunque en tan sólo cuatro piezas tumorales, el crecimiento de las células provenientes del meningioma al ser estimuladas por progesterona (43). Posteriormente el implante de tejido tumoral en ratones sin timo reveló un efecto inhibidor del crecimiento celular por parte del meningioma al ser éste sometido al RU38486 (RU486) (41). Entretanto Olson y colaboradores y posteriormente Blankestein y colaboradores demostraban similares consecuencias con el uso in vitro del RU $486(17,49)$.

No obstante que el acetato de medroxiprogesterona había demostrado unirse a receptores de progesterona (40) reducía las fracciones de crecimiento de los meningiomas en vivo (39), en un estudio clínico en casos de meningiomas irresecables y usando dosis de 40-80 $\mathrm{mg}$ de megestrol cada 6 horas por periodo entre 1-12 meses no se pudo demostrar la eficacia del mencionado agente al no evidenciarse respuestas tumorales y por alto grado de toxicidad sistémica siendo el principal de estos el aumento de peso corporal que fue hasta de $14 \mathrm{~kg}$ (56).

El uso del antiprogestágeno mifepristone o RU486 aparece como una opción interesante a la luz de trabajos clínicos publicados como los de Grunberg y colaboradores (9) y Lamberts (34) que de manera independiente demuestran respuestas objetivas en pacientes sometidos a tratamiento con mifepristone en dosis de $200 \mathrm{mg} / \mathrm{d}$ por un año y con efectos colaterales leves como fatiga, náuseas, vómitos, ginecomastia y cesación de las menstruaciones. Entre las respuesta objetivas el tratamiento encontramos disminución del tamaño tomográfico de las lesiones tumorales y mejora en el examen de campo visual. Por otro lado una mejoría de orden subjetivo también se evidenció de la cefalea. Si bien es cierto estas series recientes reúnen pocos casos, los resultados no dejan de ser importantes y ameritan estudios más amplios.

De allí la determinación de la presencia de receptores de progesterona de manera rápida (2 horas de procesamiento total) y en tejidos fijados previamente en parafina mediante el uso de anticuerpos monoclonales permiten de manera rápida y sin la necesidad de contar con tejido recientemente extirpado, reunir retrospectivamente paciente con esta patología lo que difícilmente se hacía antes cuando sólo se estudiaban casos recientemente operados. Esto daría la posibilidad a un número mayor de pacientes que habiendo sido intervenidos en el pasado que sean positivos para receptores de progesterona y que por razones de 
irresecabilidad, recurrencia o alto riesgo no puedan gozar del beneficio de la re-intervención puedan gozar de los beneficios de la hormonoterapia con mifepristone. Resultaría interesante el determinar si las tasas de recurrencia disminuyen con el uso de RU 486 así como el evaluar clínicamente su uso por mayor tiempo e inclusive se podría estudiar la posibilidad de tornar resecables algunos tumores irresecables dada la observación en la reducción del tamaño tumoral.

En conclusión este trabajo demuestra la presencia de receptores de progesterona en un 37\% de la muestras estudiadas mediante el uso de técnicas de inmunohistoquímica especialmente diseñadas para ser empleadas en tejido previamente fijado en parafina. Creemos importante el realizar estudios de orden clínico y terapéutico mediante el uso de antiprogestágenos como el mifepristone o RU 486, que a la luz de últimos estudios ha mostrado efectos beneficiosos y que por tanto permitan sistematizar la determinación de los receptores de progesterona con fines de una intervención médica.

\section{Agradecimiento:}

Al Instituto Nacional de Enfermedades Neoplásicas por las facilidades para la realización de este trabajo y muy especialmente al Dr. Hugo Heinicke del Dpto. de Neurocirugía por su invalorable contribución.

Al Dr. Alejandro Rosell por su asesoría permanente y desinteresada. Dedico este esfuerzo a mis padres y a la memoria del Dr. Serbauste.

\section{REFERENCIAS BIBLIOGRAFICAS}

1. Longstreth WT, Lelie K. Epidemiology of intracranial meningiomas. Cancer 1993; 72 : 639-648.

2. Greenberg M. Handbook of Neurosurgery. Third Edition. Tampa Florida: Greenberg graphics Inc; 1994.

3. Sheehy J. and Crockard A. Multiple meningiomas: A long term review. J Neurosurg 1983; 59: $1-5$.

4. Black M. Brain tumors. N Engl J Med 1991; 324: 1555-1562.

5. Le May D, Bucci M, Farhat SM. Malignant Transformation of recurrent meningioma with pulmonary metastases. Surg Neurol 1989; 31: 365-8.

6. Vakiti R, Muller J. Intracytoplasmatic Lumina in meningiomas: An ultrastructural and Immunohistological Study. Neurosurgery 1988; 23: 180-184.

7. Nayef RF, Laws RE. Meningioma: A historical study of the tumor and its surgical management. Neurosurgery 1990; 26: 832-847.

8. Rubinstein B, Shein M, Reichenthal E. The association of carcinoma of the breast with meningioma. Surg Gynecol and Obstet 1989; 169: 334-336.

9. Grunber M, Weiss M.. Treatment of unresectable meningiomas with the antiprogesterone agent mifepristone. J Neurosurg 1991; 74: 861-866. 
10. Kaper J.. Effects of progesterone on the Response to Epidermal Growth Factor and other Growth Factors in cultured human meningioma cells. Cancer Res 1990; 50: 2604-2607.

11. Kurihara M, Tokunaga Y. Characterization of insulin like growth factor receptors in meningiomas. J Neurosurg 1989; 71: 538-544.

12. Liang SL, Chin W. et al. Steroid hormone receptors in meningiomas of Chinese patients. Neurosurgery $1989 ; 25: 541-545$.

13. Halper J. Estrogen and progesterone receptors in meningiomas. Comparison of nuclear Binding, Dextran Coated charcoal, and Inmunoperoxidase Staining Assays. Neurosurgery 1989; 25: 546-553.

14. Maiuri F, Montagnani S. Oestrogen and progesterone sensitivity in cultured meningioma cells. Neurological Research 1989; 11: 9-13.

15. Kornblum A.. Steroid Receptors in human brain and spinal cord tumors. Neurosurgery 1989; 23: 185-188.

16. Grunberg S. et al. Correlation of meningioma hormone receptor status with hormone sensitivity in a tumor stem cell assay. J Neurosurg 1987; 66: 405-408.

17. Whittle IR. Et al. Sex hormone receptor in intracranial tumors and normal brain. Eur J Surg Oncol 1987; 13: 303-307.

18. Cahill D. Estrogen and progesterone receptors in meningiomas. J Neurosurg 1984; 60: 985-993.

19. Gabos S, Berkel J. Meta Analysis of progestin and estrogen receptors in human meningiomas. Neuroepidemiology 1992; 11: 255-260.

20. Koehorst SG. Aberrant estrogen receptors species in human meningioma tissue. J Steroid Biochem Molec Biol 1992; 43: 57-61.

21. Kostron H, Daxenbichler G. Steroidrezeptoren and atypische Histologie als prognostische Parameter bei Meningiomen. Wien - Klin Wochenschr 1990; 102: 525-8.

22. Klaus P, Silke G. Estrogen receptor immunoreactivity in meningiomas. J Neurosurg 1987; 67: 237-243.

23. Blankestein MA, Meulen-Dijk VD. Assay of oestrogen and progestin receptors in human meningioma cytosols using immunological methods. Clinica Chimica Acta 1987; 165: 189195.

24. Carroll SR, Glowacka D. Progesterone receptor expression in meningiomas. Cancer Res 1993, 53: 1312-1316.

25. Maxwell M. Expression of androgen and progesterone receptors in primary human meningiomas. J Neurosurg 1993; 78: 456-462.

26. Perrot Applanat M, Kujas M. Inmunocytochemical Study of Progesterone Receptor in human meningiomas. Acta Neurochir (Wien) 1992; 115: 20-30.

27. Piantelli M. Type II Estrogen Binding Sites and Antiproliferative Activity of Quercetin in human meningiomas. Cancer 1993; 71: 193-8. 
28. Piquer J, Cerda M, Llusch A. Correlations of female steroid receptors with histologic features in meningiomas. Acta Neruochir (Wien) 1991; 110: 38-43.

29. Ranka RS. Sex steroid receptors in intracranial tumors. Cancer 1990; 65: 1968-1970.

30. Waelti ER. Inmunocytochemical evidence of progesterone receptors in human meningiomas. Surg Neurol 1989; 3: 172-6.

31. Tilzer L. Steroid receptor proteins in human meningiomas. Cancer 1982; 49: 633-636.

32. Jaaskelainen J. The growth rate of intracranial meningiomas and its relation to Histology. Surg Neurol 1985; 24: 165-172.

33. Sidney M. Recurrence of meningiomas after operation. Surg Neurol 1986; 25: 436-440.

34. Lamberts SWJ, Tanghe HLJ, Avezaat CJJ, et al. Mifepristone (RU 486) treatment of meningiomas. Journal Neurol Neurosurg and Psychiatry 1992; 55: 486-490.

35. Schrell U, Adams E. Hormonal dependency of cerebral meningiomas. Part I. J Neurosurg 1990; 73: 743-749.

36. Benzel E, Gelder F. Correlation between sex hormone binding and peritumoral edema in intracranial meningiomas. Neurosurgery 1988; 23: 169-174.

37. Waelti E. Endocrine Manipulation of meningioma with medroxiprogesterone acetate. Surgery Neurol 1989; 31: 96-100.

38. Blankenstein MA. Effect steroids and antisteroids on human meningioma cells in primary cuture. J Steroid Biochem 1989; 34: 419-421.

39. Markwalder T. Hormonotherapy of meningioma with medroxiprogesterone acetate. Surg Neurol 1988; 30: 97-101.

40. Markwalder T. Endocrine manipulation of meningiomas with medroxiprogesterone acetate. Surg Neurol 1987; 28: 3-9.

41. Olson J. Effect of antiprogesterone RU 38486 on meningioma implanted into nude mice. J Neurosurg 1987; 66: 584-587.

42. Olson J. Hormonal manipulation of meningiomas in vitro. J Neurosurg 1986; 65: 99-107.

43. Jay R. J. Modulation of meningioma cell growth by sex steroid hormones in vitro. J Neurosurg 1985; 62: 757-762.

44. Courriere P. Hormonal steroid receptors in Intracranial Cancer Oncol 1985; 21: 711-714.

45. Barnett HG. Posttraumatic Intracranial Meningioma: A Case Report and Review of the Literature. Neurosurgery 1986; 18: 75-78.

46. Perrot-Applanat M. et al. Inmunocitochemical Study with monoclonal antibodies to progesterone receptor in human breast tumors. Cancer Res 1987; 47: 2652-2661.

47. Reiner A. Inmunocitochemical Study with monoclonal antibodies to progesterone receptor in human breast tumors. Cancer Res 1990. 50: 7057-7061.

48. Press MF. Localization of Progesterone Receptor with Monoclonal Antibodies to the 
Human Progestin Receptor. Endocrinology 1988; 122: 1165-1175.

49. Lesch KP. Estrophilin Immunoreactivity versus Estrogen Receptor Binding Activity in Meningiomas: Evidence for Multiple Estrogen Binding Sites. Surg. Neurol 1987; 28: 181188.

50. Lesch KP. Simultaneous Estradiol and Progesterone Receptor Analysis in Meningiomas. Surg Neurol 1986; 26: 257-63.

51. Adam E. Hormonal dependency of cerebral meningiomas. Part II. J Neurosurgery 1990; 73: $750-755$.

52. Tjeerd WA, Hervé LJ. Progesterone, oestradiol, human meningiomas and their CT characteristics. Eur J Cancer 1991; 27: 1453-1457.

53. Meixensberger J, Caffier H, Naumann M, et al. Sex hormone binding and peritumoral oedema in meningiomas. Is there a correlation?. Acta Neurochir (Wien) 1992; 115: 98-102.

54. Go K. Peritumoral brain edema associated with meningiomas. Neurosurgery 1989; 23 : 175-179.

55. Martuza LR. Estrogen and progestin binding by cytosolic and nuclear fractions of human meningiomas. J Neurosurg 1985; 62: 750-756.

56.Grunberg SM, Weiss M. Lack of efficacy of megestrol acetate in the treatment of unresectable meningioma. J Neuro-Oncol 1990; 8: 61-65.

\section{Correspondencia:}

Dr. Herminio Ojeda Di Ninno.

Av. Pershing 960. San Isidro, Lima, Perú 